\title{
Approximation by Brownian motion for Gibbs measures and flows under a function
}

\author{
MANFRED DENKER AND WALTER PHILIPP \\ Institut für Mathematische Stochastik, Universität Göttingen, Lotzestr. 13 D-3400 \\ Göttingen, West Germany; Department of Mathematics, University of Illinois, \\ Urbana, IL 61801, USA
}

(Received 5 August 1983)

Abstract. Let $\left\{S_{\tau}: \tau \in \mathbb{P}\right\}$ denote a flow built under a Hölder-continuous function $l$ over the base $(\Sigma, \mu)$ where $\Sigma$ is a topological Markov chain and $\mu$ some ( $\psi$-mixing) Gibbs measure. For a certain class of functions $f$ with finite $2+\delta$-moments it is shown that there exists a Brownian motion $B(t)$ with respect to $\mu$ and $\sigma^{2}>0$ such that $\mu$-a.e.

$$
\sup _{0 \leq u<I(x)}\left|\int_{0}^{t} f S_{\tau} d \tau-B\left(\sigma^{2} t\right)\right| \ll t^{\frac{1}{2}-\lambda} \quad \text { for some } 0<\lambda<\delta / 588 .
$$

One can also approximate in the same way by a Brownian motion $B^{*}(t)$ with respect to the probability $\left(\int l d \mu\right)^{-1} l d \mu$. From this, the central limit theorem, the weak invariance principle, the law of the iterated logarithm and related probabilistic results follow immediately. In particular, the result of Ratner ([6]) is extended.

\section{Introduction}

Let $\Sigma$ be a topologically mixing Markov chain with shift transformation $T$. Let $\Phi: \Sigma \rightarrow \mathbb{R}$ be a function of bounded variation satisfying $\operatorname{var}_{k} \Phi \leq b \alpha^{k}(k \geq 1 ; b>0$, $0<\alpha<1$ ) where

$$
\operatorname{var}_{k} \Phi:=\sup \left\{|\Phi(x)-\Phi(y)|: x_{i}=y_{i} \text { for all }|i| \leq k\right\} .
$$

Let $\mu$ denote the Gibbs measure given by $\Phi$. Then $\mu$ is the unique shift invariant probability measure satisfying

$$
c_{1} \leq \frac{\mu\left\{y: y_{i}=x_{i} \text { for all } i=0, \ldots, m-1\right\}}{\exp \left(-P m+\sum_{0 \leq k<m} \Phi\left(T^{k} x\right)\right)} \leq c_{2}, \quad x \in \Sigma, m \geq 1
$$

for some constants $P$ and $c_{1}>0$ and $c_{2}>0$ (see [2], [8], [10]). We shall consider the flow $\left\{S_{t}: t \in \mathbb{R}\right\}$ built under a function $l$ with base transformation $(\Sigma, T, \mu)$. In order to provide an adequate setting for the formulation of the theorem below we embed $\Sigma$ in $\Sigma_{1}:=\Sigma \times[0,1]$, the product $\sigma$-field, probability measure $\mu \times \lambda$ and the transformation $T_{1}$ defined by $T_{1}(x, \omega)=(T x, \omega)(x \in \Sigma, 0 \leq \omega \leq 1)$. Then the flow built under the function $l$ is embedded in the flow built under the function $l_{1}(x, \omega)=$ $l(x)(x \in \Sigma, 0 \leq \omega \leq 1)$ and base transformation $T_{1}$. Whenever we consider the flow $\left\{S_{t}: t \in \mathbb{R}\right\}$ together with a Brownian motion it is assumed that $\left\{S_{t}\right\}$ is embedded in the larger space. This is necessary in order to ensure the existence of a function 
independent of the process $f \circ S_{t}$ under consideration. From now on, however, we drop the index 1 to simplify the notation and because the embedding procedure is only a minor technicality. We assume that $l$ is Hölder-continuous with some exponent $\rho>0$ and that $l$ is bounded away from 0 . Denote by

$$
X:=\{(x, s): x \in \Sigma, 0 \leq s<l(x)\}
$$

the space on which $\left\{S_{t}\right\}$ is defined and by $\nu$ the probability measure on $X$ given by

$$
d \nu=\left(\int l d \mu\right)^{-1} d s \times d \mu
$$

THEOREM. Let $f$ be a measurable function defined on $X$ with

$$
E f:=\int_{X} f d \nu=0
$$

and

$$
E|f|^{2+\delta}:=\int_{X}|f|^{2+\delta} d \nu<\infty
$$

for some $0<\delta \leq 1$. Suppose that

$$
E\left|f-E\left(f \mid(\gamma)_{-n}^{n}\right)\right|^{2+\delta} \ll n^{-(2+(7 / \delta))(2+\delta)}
$$

where $(\gamma)_{-n}^{n}$ denotes the $\sigma$-field generated by the atoms of the form $\{(x, s): x \in A$, $0 \leq s<l(x)\}$ and where $A$ is a centred cylinder of length $2 n+1$. Then

$$
\sigma^{2}:=\lim _{t \rightarrow \infty} t^{-1} E\left(\int_{0}^{t} f\left(S_{\tau}(w)\right) d \tau\right)^{2}
$$

exists. Moreover, if $\sigma^{2}>0$ then there exists a standard Brownian motion $\{B(t, x)$ : $t \geq 0, x \in \Sigma\}$, defined on $(\Sigma, \mu)$ such that

$$
\sup _{0 \leq u<l(x)}\left|\int_{0}^{t} f\left(S_{\tau}(x, u)\right) d \tau-B\left(\sigma^{2} t, x\right)\right| \ll t^{\frac{1}{2}-\lambda}
$$

for $\mu$-almost all $x$ and some $0<\lambda<\delta / 588$. Moreover, (2) remains valid if we replace the Brownian motion $B$ by a standard Brownian motion $\left\{B^{*}(t, x): t \geq 0, x \in \Sigma\right\}$ defined on $\left(\Sigma, \mu^{*}\right)$ where $d \mu^{*}(x):=\left(\int l d \mu\right)^{-1} l(x) d \mu(x)$.

Remark. Here and throughout this paper we use the Vinogradov symbol « instead of Landau's ' $O$ ' notation. Thus, $g \ll h$ means $g=O(h)$.

This theorem implies the usual corollaries such as the central limit theorem, the law of the iterated logarithm, upper and lower class results for the law of the iterated logarithm, the functional versions of the central limit theorem and the law of the iterated logarithm, etc. We shall now list three of these results, which hold under the hypothesis of the theorem.

COROllaRy 1 (The central limit theorem). We have for all $z \in \mathbb{R}$ :

$$
\lim _{t \rightarrow \infty} \nu\left(\left\{w: \frac{1}{\sigma \sqrt{t}} \int_{0}^{t} f\left(S_{\tau} w\right) d \tau \leq z\right\}\right)=\frac{1}{\sqrt{2 \pi}} \int_{-\infty}^{z} \exp \left(-\frac{1}{2} u^{2}\right) d u
$$


COROLlary 2 (The law of the iterated logarithm). We have for $\nu$-almost all $w$ :

$$
\limsup _{t \rightarrow \infty}\left(2 \sigma^{2} t \log \log t\right)^{-\frac{1}{2}} \int_{0}^{t} f\left(S_{\tau} w\right) d \tau=1 .
$$

COROLlaRY 3 (The weak invariance principle). Let

$$
\zeta_{n}(t):=\sigma^{-1} n^{-\frac{1}{2}} \int_{0}^{n t} f \circ S_{\tau} d \tau \quad(0 \leq t \leq 1, n \in \mathbb{N}) .
$$

Then $\left\{\zeta_{n}: n \geq 1\right\}$ converges weakly in $C([0,1])$ to the standard Wiener measure.

All results of this type can easily be reduced to well-known theorems on functions of mixing sequences of random variables. This is also the method we shall use in $\S 3$ to prove the theorem. For instance, relation (11) of [6] combined with the classical results of Ibragimov [4] and Billingsley [1, theorem 20.2 and pp. 194/195] and a standard result (see e.g. $[7$, p. 390]) immediately yields corollary 1 under the assumption $\delta>0$, thus extending [6] in various directions.

\section{A moment estimate}

Let $\left\{\xi_{n}, n \in \mathbb{Z}\right\}$ be a sequence of random variables. For $a<b$ let $\mathscr{F}_{a}^{b}$ be the $\sigma$-field generated by $\xi_{a}, \xi_{a+1}, \ldots, \xi_{b}$. The sequence $\left\{\xi_{n}, n \in \mathbb{Z}\right\}$ is called $\varphi$-mixing if there exists a sequence of real numbers $\varphi(n) \rightarrow 0$ such that

$$
|P(A \cap B)-P(A) P(B)| \leq P(A) \varphi(n)
$$

for all $A \in \mathscr{F}_{-\infty}^{k}, B \in \mathscr{F}_{k+n}^{\infty}$ and all $k \in \mathbb{Z}, n \geq 1$. Next, let $g$ be a measurable mapping from the space of doubly infinite sequences $\left(\ldots, \alpha_{-1}, \alpha_{0}, \alpha_{1}, \ldots\right)$ of real numbers into the real line. For $\xi=\left(\ldots, \xi_{-1}, \xi_{0}, \xi_{1}, \ldots\right)$ and for the shift $T$ put

$$
\eta_{n}=g\left(T^{n} \xi\right), \quad n \geq 1
$$

and

$$
\eta_{m n}=E\left(\eta_{n} \mid \mathscr{F}_{n-m}^{n+m}\right), \quad m, n \geq 1 .
$$

LEMMA 1. Let $\left\{\xi_{n} ; n \in \mathbb{Z}\right\}$ be a stationary $\varphi$-mixing sequence of random variables with

$$
\sum \varphi^{\frac{1}{2}}(n)<\infty
$$

and let $\eta_{n}$ and $\eta_{m n}$ be defined by (3) and (4). Assume for $g$ and $\left\{\xi_{n} ; n \in \mathbb{Z}\right\}$ that $E \eta_{1}=0$ and that for some $0<\delta \leq 1, E\left|\eta_{1}\right|^{2+\delta}<\infty$ and

$$
\left\|\eta_{1}-\eta_{m 1}\right\|_{2+\delta} \ll m^{-2} \quad \text { as } m \rightarrow \infty \text {. }
$$

Then

$$
E\left|\sum_{j \leq n} \eta_{j}\right|^{2+\delta} \ll n^{1+(\delta / 2)}
$$

where the constant implied by $\ll$ depends only on $\delta,\{\varphi(n), n \geq 1\}, E\left|\eta_{1}\right|^{2+\delta}$ and the constant implied by $\ll$ in (6).

Remark. It is easy to see that lemma 1 remains valid for $\delta=0$. Indeed by [1, p. 185], Hölder's inequality and (6) - actually the proof of (8) below will also do -

$$
\left|E \eta_{1} \eta_{k+1}\right| \ll \varphi^{\frac{1}{2}\left(\frac{1}{3} k\right)+k^{-2}} \text {. }
$$


Thus by stationarity and (5)

$$
E\left(\sum_{j \leq n} \eta_{j}\right)^{2}=\sum_{j \leq n} E \eta_{j}^{2}+2 \sum_{1 \leq j<n}(n-j) E \eta_{1} \eta_{j+1} \ll n .
$$

The proof of lemma 1 is a minor modification of the proof of lemma 7.4 of $[3, \mathrm{pp}$. 225-227]. For some reader's convenience we present the proof in some detail. Let

and

$$
k:=3\left[n^{\frac{1}{2}}\right]
$$

$$
s_{n}:=\sum_{j=1}^{n} \eta_{j}, \quad t_{n}:=\sum_{j=n+1}^{n+k} \eta_{j}, \quad \hat{s}_{n}:=\sum_{j=n+k+1}^{2 n+k} \eta_{j}, \quad c_{n}:=E\left|\sum_{j=1}^{n} \eta_{j}\right|^{2+\delta} .
$$

We first show that

$$
E\left|s_{n}\right|^{u}\left|\hat{s}_{n}\right|^{v} \leq n^{1+\frac{1}{2} \delta}+o\left(c_{n}\right)
$$

where $u+v=2+\delta$ and $u=1,2, \delta$ or $1+\delta$. To prove (8) we put $m:=\frac{1}{3} k$ and introduce the sums

$$
s_{m n}:=\sum_{j \leq n} \eta_{m j} \quad \text { and } \quad \hat{s}_{m n}:=\sum_{j=n+k+1}^{2 n+k} \eta_{m j}
$$

Then

$$
\begin{aligned}
E\left|s_{n}\right|^{u}\left|\hat{s}_{n}\right|^{v} \ll & E\left|s_{n}-s_{m n}\right|^{u}\left|\hat{s}_{n}-\hat{s}_{m n}\right|^{v}+E\left|s_{n}-s_{m n}\right|^{u}\left|\hat{s}_{m n}\right|^{v} \\
& +E\left|s_{m n}\right|^{u}\left|\hat{s}_{n}-\hat{s}_{m n}\right|^{v}+E\left|s_{m n}\right|^{u}\left|\hat{s}_{m n}\right|^{v} \\
= & \mathrm{I}+\mathrm{II}+\mathrm{III}+\mathrm{IV}, \text { say. }
\end{aligned}
$$

By Hölder's inequality, Minkowski's inequality, stationarity and (6)

$$
\mathrm{I} \leq E\left|s_{n}-s_{m n}\right|^{2+\delta} \ll\left(n \cdot m^{-2}\right)^{2+\delta} \ll 1 .
$$

Hence by Minkowski's inequality, stationarity and since $v \leq 2$

$$
\begin{aligned}
\mathrm{II} & \leq\left\|s_{n}-s_{m n}\right\|_{2+\delta}^{u}\left\|\hat{s}_{m n}\right\|_{2+\delta}^{v} \ll\left\|\hat{s}_{m n}-\hat{s}_{n}\right\|_{2+\delta}^{v}+\left\|\hat{s}_{n}\right\|_{2+\delta}^{v} \\
& \ll 1+c_{n}^{2 /(2+\delta)}=o\left(c_{n}\right) .
\end{aligned}
$$

Likewise

$$
\text { III }=o\left(c_{n}\right) .
$$

For the estimate of IV we use Ibragimov's lemma [4, lemma 1.1]: Let $\xi$ and $\eta$ be measurable $\mathscr{F}_{1}^{k}$ and $\mathscr{F}_{k+n}^{\infty}$ respectively. If $1 / p+1 / q=1$ and $E|\xi|^{p}<\infty$ and $E|\eta|^{q}<\infty$ then

$$
|E \xi \eta-E \xi E \eta| \leq 2(\varphi(n))^{1 / p}\|\xi\|_{p}\|\eta\|_{q} .
$$

We apply this lemma with $p=(2+\delta) / u$ and $q=(2+\delta) / v$ and obtain by Minkowski's inequality, Hölder's inequality, stationarity, (9), since by (7), $k-2 m=\left[n^{\frac{1}{2}}\right]$, and since by the above remark lemma 1 holds for $\delta=0$,

$$
\begin{aligned}
\mathrm{IV} & \leq E\left|s_{m n}\right|^{u} E\left|s_{m n}\right|^{v}+2(\phi(k-2 m))^{\delta /(2+\delta)} E\left|s_{m n}\right|^{2+\delta} \\
& \leq E\left|s_{n}\right|^{u} E\left|s_{n}\right|^{0}+o\left(c_{n}\right) \leq\left(E s_{n}^{2}\right)^{1+\frac{1}{2} \delta}+o\left(c_{n}\right) \ll n^{1+\frac{1}{2} \delta}+o\left(c_{n}\right) .
\end{aligned}
$$

We add the estimates for I, II, III and IV and obtain (8). Consequently, for some constant $a_{1}$,

$$
\begin{aligned}
E\left|s_{n}+\hat{s}_{n}\right|^{2+\delta} & \leq E\left\{\left|s_{n}+\hat{s}_{n}\right|^{2}\left(\left|s_{n}\right|^{\delta}+\left|\hat{s}_{n}\right|^{\delta}\right)\right\} \\
& \leq c_{n}(2+o(1))+a_{1} n^{1+\frac{1}{2} \delta} .
\end{aligned}
$$


This corresponds to [3, (7.8)]. We pick up the proof of [3, lemma 7.4] on p. 226 just before [3, (7.11)] and follow it to its end replacing $m$ by 2 and $g\left(x_{j}\right)$ by $\eta_{j}$.

\section{Proof of theorem}

For the proof of the theorem we apply the following proposition (see $[5$, p. 81 , remark]).

PROPOSITION 1. Let $\left\{\xi_{n}, n \in \mathbb{Z}\right\}$ be a sequence of random variables satisfying a strong mixing condition

$$
\beta(n):=\sup \left\{|P(A \cap B)-P(A) P(B)|: A \in \mathscr{F}_{-\infty}^{t}, B \in \mathscr{F}_{t+n}^{\infty}\right\} \ll n^{-168(1+(2 / \delta))} .
$$

Let $g$ be a measurable mapping from the space of doubly infinite sequences $\left(\ldots, \alpha_{-1}, \alpha_{0}, \alpha_{1}, \ldots\right)$ of real numbers into the real line. Let $\eta_{n}=$ $g\left(\ldots, \xi_{n-1}, \xi_{n}, \xi_{n+1}, \ldots\right), n=1,2, \ldots$, and $\eta_{m n}=E\left(\eta_{n} \mid \mathscr{G}_{n-m}^{n+m}\right)$, where $\mathscr{G}_{a}^{b}$ is any collection of sub $\sigma$-fields such that $\mathscr{G}_{a}^{b} \subset \mathscr{F}_{a}^{b}$ for all $a \leq b$. We assume of the function $g$ and the sequence $\left\{\xi_{n}, n \in \mathbb{Z}\right\}$ that

$$
E \eta_{n}=0 \quad n=1,2, \ldots
$$

and that there exist constants $0<\delta \leq 2$ and $c>0$ such that

$$
E\left|\eta_{n}\right|^{2+\delta} \leq c,
$$

and

$$
\left\|\eta_{n}-\eta_{m n}\right\|_{2+\delta} \leq \mathrm{cm}^{-(2+(7 / \delta))}
$$

for all $n, m=1,2,3, \ldots$ Moreover, suppose that

$$
E\left(\sum_{n \leq N} \eta_{n}\right)^{2}=\tau N+O\left(N^{1-(\delta / 30)}\right)
$$

for some $\tau>0$. Then without changing its distribution we can redefine the sequence $\left\{\eta_{n}, n \geq 1\right\}$ on a richer probability space on which there exists a standard Brownian motion $\{B(t), t \geq 0\}$ such that with probability 1

$$
\sum_{n \leq t} \eta_{n}-B(\tau t) \ll t^{\frac{1}{2}-\lambda} \quad \text { as } t \rightarrow \infty
$$

for any $\lambda<\delta / 588$.

Let

$$
F(x)=\int_{0}^{l(x)} f(x, s) d s, \quad x \in \Sigma,
$$

and let $n(\tau, x)$ be the largest integer $r$ such that

$$
\sum_{0 \leq i \leq r} l\left(T^{i} x\right)<\tau .
$$

LEMMA 2. We have for all $u$ and $\mu$-almost all $x$ as well as in $L^{2}$

$$
\int_{0}^{t} f\left(S_{\tau}(x, u)\right) d \tau-\sum_{0 \leq i \leq n(t, x)} F\left(T^{i} x\right) \ll 1 .
$$


Proof. Put

$$
\begin{aligned}
\mathrm{I} & :=\left|\int_{0}^{t} f\left(S_{\tau}(x, u)\right) d \tau-\int_{0}^{t} f\left(S_{\tau}(x, 0)\right) d \tau\right|, \\
\mathrm{II} & :=\left|\sum_{0 \leq i \leq n(t, x)} \int_{0}^{l\left(T^{i} x\right)} f\left(T^{i} x, s\right) d s-\sum_{0 \leq i \leq n(t, x)} F\left(T^{i} x\right)\right|, \\
\mathrm{III} & :=\int 1\left\{l\left(T^{n(t, x)} x\right) \leq \tau \leq t\right\}\left|f\left(S_{\tau}(x, 0)\right)\right| d \tau .
\end{aligned}
$$

Then the left-hand side in the lemma is bounded by I + II + III. Now II =0 by (14). To estimate I we observe that

$$
\mathrm{I} \leq 2 \int_{0}^{c}\left|f\left(S_{\tau}(x, u)\right)\right| d \tau, \quad c:=\max \{|l(x)|: x \in \Sigma\}
$$

since $l$ is bounded away from 0 and $\infty$. Now

$$
\begin{aligned}
\iint_{0}^{c}\left|f\left(S_{\tau}(x, u)\right)\right|^{2} d \tau d \mu(x) & =\iint_{0}^{c}\left|f\left(S_{\tau+u}(x, 0)\right)\right|^{2} d \tau d \mu(x) \\
& \ll \sum \iint_{0}^{l\left(T^{i} x\right)}\left|f\left(S_{\tau}\left(T^{i} x, 0\right)\right)\right|^{2} d \tau d \mu(x)<\infty
\end{aligned}
$$

since $E|f|^{2}<\infty$ and the above sum is finite. Hence I is bounded for $\mu$-almost all $x$ and all $u$. In the same way one can see that III is also bounded.

Because of lemma 2 the approximation of the integral has been reduced to the approximation of a random sum of random variables by a Brownian motion. Proposition 1 specialized to the present situation will yield the approximation of the sum $\sum_{i \leq t} F\left(T^{i} x\right)$ by a Brownian motion. But because of lemma 3 below the passage to the random sum will be easy.

For the proof of lemma 3 as well as for the application of proposition 1 we first set up the basic probability structure. The underlying probability space is $(\Sigma, \mu)$. Let $\tilde{\gamma}$ be the natural generator of the topological Markov chain $\Sigma$. As has been observed by Ratner [6, p. 182] the process $(T, \tilde{\gamma})$ satisfies a mixing condition somewhat stronger than $\varphi$-mixing with an exponential rate of decay. This was obtained from Sinai's [10] construction of invariant Gibbs measures of transitive C-flows of class $C^{2}$. However, much more is true. Using Ruelle's Perron-Frobenius theorem (cf. [8]), Bowen obtained [2, p. 24] the following weak dependence relation:

$$
|\mu(A \cap B)-\mu(A) \mu(B)| \leq K \beta^{n} \mu(A) \mu(B)
$$

for all $A \in(\tilde{\gamma})_{-m}^{0}, B \in(\tilde{\gamma})_{n}^{n+k}$ and all $m, k, n \geq 0$. Here $K$ and $\beta \in(0,1)$ are constants. This condition is known in the literature as $\psi$-mixing and is obviously more restrictive than $\varphi$-mixing, which in turn is more restrictive than strong mixing. Let $\xi_{n}$ be the $n$th coordinate map of $\Sigma$. Then $\left\{\xi_{n}, n \in \mathbb{Z}\right\}$ satisfies a strong mixing condition with exponentially fast mixing rate $\beta(n)$.

LEMMA 3. For any $\varepsilon>0$ we have for $\mu$-almost all $x$ :

Here $\bar{l}:=\int l d \mu=E_{\mu} l$.

$$
n(t, x)-t / \bar{l} \ll t^{\frac{1}{2}+\varepsilon} .
$$


Proof. Define

$$
\rho_{n}(x):=l\left(T^{n} x\right)-\bar{l} \quad x \in \Sigma .
$$

Since $l$ is Hölder continuous we have for all $m \geq 1$

$$
\left\|l-E_{\mu}\left(l \mid(\tilde{\gamma})_{-m}^{m}\right)\right\|_{2} \leq K \alpha^{m}
$$

for some constants $K$ and $\alpha \in(0,1)$. Thus setting

we obtain

$$
\rho_{m n}:=E_{\mu}\left(\rho_{n} \mid(\tilde{\gamma})_{n-m}^{n+m}\right)
$$

$$
\left\|\rho_{n}-\rho_{m n}\right\|_{2} \leqslant K \alpha^{m} \text {. }
$$

Hence by lemma 1 , applied with $\delta=0$ we have for all $M \geq 0, N \geq 1$

$$
E\left(\sum_{i=M+1}^{M+N} \rho_{i}\right)^{2} \ll N
$$

We apply the Gaal-Koksma strong law of large numbers (see e.g. [5, theorem A1, p. 134]) and obtain for each $\varepsilon>0$

$$
\sum_{i \leq n}\left(l\left(T^{i} x\right)-\bar{l}\right) \ll n^{\frac{1}{2}+\varepsilon}
$$

for $\mu$-almost all $x$. For $r \geq 1$ put

$$
\left.A_{r}:=\left\{x: \mid \sum_{i \leq n}\left(l\left(T^{i} x\right)\right)-\bar{l}\right) \mid \leq n^{\frac{1}{2}+\varepsilon}, \quad \text { for all } n \geq r\right\} .
$$

If for some $r \geq 1$ we have $x \in A_{r}$ then for all $n \geq r$

$$
t_{1}:=n \bar{l}-n^{\frac{1}{2}+\varepsilon} \leq \sum_{i \leq n} l\left(T^{i} x\right) \leq t_{2}:=n \bar{l}+n^{\frac{1}{2}+\varepsilon} .
$$

Thus $n\left(t_{1}, x\right) \leq n$ and $n\left(t_{2}, x\right) \geq n$. Let $t$ be given and let $m$ and $n$ be such that $n \bar{l}+n^{\frac{1}{2}+\varepsilon} \leq t \leq m \bar{l}-m^{\frac{1}{2}+\varepsilon}$ and $m \bar{l}-m^{\frac{1}{2}+\varepsilon} \leq n \bar{l}+n^{\frac{1}{2}+\varepsilon}+O(1)$. Then we obtain by elementary manipulations

$$
t^{-1} n(t, x)-1 / \bar{l} \ll t^{-\frac{1}{2}+\varepsilon} .
$$

We put $\eta_{n}(x):=F\left(T^{n} x\right), x \in \Sigma$. We now apply proposition 1 to the sequence $\left\{\eta_{n}, n \geq 1\right\}$. Since

$$
E \eta_{0}=\int F(x) d \mu(x)=\iint_{0}^{l(x)} f(x, s) d s d \mu(x)=\bar{l} E f=0
$$

and likewise

$$
\int|F(x)|^{2+\delta} d \mu(x) \leq \bar{l} E|f|^{2+\delta}
$$

conditions (10) and (11) are satisfied. We now verify (12). We have

where

$$
E_{\mu}\left|F-E_{\mu}\left(F \mid(\tilde{\gamma})_{-n}^{n}\right)\right|^{2+\delta} \leq 8(\mathrm{I}+\mathrm{II})
$$

$$
\begin{aligned}
\mathrm{I} & :=E_{\mu}\left|\int_{0}^{l(x)} f(x, s) d s-\int_{0}^{l(x)} E\left\{f \mid(\gamma)_{-n}^{n}\right\}(x, s) d s\right|^{2+\delta} \\
& \leq \iint_{0}^{l(x)}\left|f(x, s)-E\left\{f \mid(\gamma)_{-n}^{n}\right\}(x, s)\right|^{2+\delta} d s d \mu(x) \\
& \leq \bar{l} \cdot E\left|f-E\left(f \mid(\gamma)_{-n}^{n}\right)\right|^{2+\delta} \ll n^{-(2+(7 / \delta))(2+\delta)}
\end{aligned}
$$


by hypothesis and

$$
\mathrm{II}:=E_{\mu}\left|E_{\mu}\left\{\int_{0}^{I(\cdot)} f(\cdot, s) d s \mid(\tilde{\gamma})_{-n}^{n}\right\}(x)-\int_{0}^{I(x)} E\left\{f \mid(\gamma)_{-n}^{n}\right\}(x, s) d s\right|^{2+\delta} .
$$

Now

$$
\begin{aligned}
&\left|E_{\mu}\left\{\int_{0}^{l(\cdot)} f(\cdot, s) d s \mid(\tilde{\gamma})_{-n}^{n}\right\}(x)-\int_{0}^{l(x)} E\left\{f \mid(\gamma)_{-n}^{n}\right\}(x, s) d s\right| \\
&=\mid \sum_{\tilde{C} \in(\tilde{y})_{-n}^{n}} \frac{1}{\mu(\tilde{C})} \int_{\tilde{C}} \int_{0}^{l(y)} f(y, s) d s d \mu(y) \cdot 1_{\tilde{C}}(x) \\
&-\int_{0}^{l(x)} \sum_{C \in(\gamma)_{-n}^{n}} \frac{1}{\nu(C)} \int_{C} f(y, \tau) d \nu(y, \tau) 1_{C}(x, s) d s \mid \\
&=\left|\sum_{\tilde{C} \in(\tilde{\gamma})_{-n}^{n}} 1{ }_{\tilde{C}}(x) \int_{C} f d \nu\left[\frac{\bar{l}}{\mu(\tilde{C})}-\frac{l(x)}{\int_{\tilde{C}} l(y) d \mu(y) / \bar{l}}\right]\right| \\
& \leq \sum_{\tilde{C} \in(\tilde{\gamma})_{-n}^{n}} 11_{\tilde{C}}(x) \frac{1}{\mu(\tilde{C})} \int_{\tilde{C}}|F| d \mu \cdot \frac{\int_{\tilde{C}}|l(y)-l(x)| d \mu(y)}{\int_{\tilde{C}} l(y) d \mu(y)} \\
& \ll \sum_{\tilde{C} \in(\tilde{\gamma})_{-n}^{n}} 1{ }_{\tilde{C}}(x) \frac{1}{\mu(\tilde{C})} \int_{\tilde{C}}|F| d \mu \cdot \alpha^{n} / \min \{l(y): y \in \Sigma\} \\
& \ll \alpha^{n} E_{\mu}\left(|F| \mid(\tilde{\gamma})_{-n}^{n}\right)(x)
\end{aligned}
$$

where $0<\alpha<1$ is as in the proof of lemma 3. Hence II decreases exponentially fast since

$$
E_{\mu}\left(E_{\mu}\left(|F| \mid(\tilde{\gamma})_{-n}^{n}\right)\right)^{2+\delta} \leq E_{\mu}|F|^{2+\delta} \leq \bar{l} E|f|^{2+\delta}<\infty
$$

Next we verify (13). Since (12) holds we have with $m=\left[\frac{1}{3} n\right]$

$$
\begin{aligned}
E \eta_{1} \eta_{n} & =E \eta_{1}\left(\eta_{n}-\eta_{m n}\right)+E \eta_{m n}\left(\eta_{1}-\eta_{1 m}\right)+E \eta_{1 m} \eta_{m n} \\
& \ll m^{-(2+(7 / \delta))}+\beta^{\frac{1}{2}}(m) \ll n^{-(2+(7 / \delta))},
\end{aligned}
$$

by (9) and since $\beta(m) \rightarrow 0$ exponentially fast. Thus

$$
\begin{aligned}
E_{\mu}\left(\sum_{i \leq n} \eta_{i}\right)^{2} & =n E \eta_{1}^{2}+2 \sum_{i=1}^{n-1}(n-i) E \eta_{1} \eta_{i+1} \\
& =n\left(E \eta_{1}^{2}+2 \sum_{i=1}^{\infty} E \eta_{1} \eta_{n+1}\right)+O(1) \\
& =n \tau+O(1), \text { say, }
\end{aligned}
$$

where $\tau \geq 0$. This proves (13).

We finally show the existence of the limit in (1) showing in fact that $\sigma^{2}=\tau / \bar{l}$. By stationarity and the $L^{2}$ statement in lemma 2 this claim will follow from

$$
E_{\mu}\left(\sum_{i \leq|n(t, x)-t / \bar{\eta}|} F\left(T^{i} x\right)\right)^{2}=o(t)
$$

We first observe that by stationarity, by lemma 1 and $[9$, theorem B]

$$
E_{\mu} \max _{m \leq n}\left|\sum_{i \leq m} F\left(T^{i} x\right)\right|^{2+\delta} \ll n^{1+\frac{1}{2} \delta}
$$


We also note that $|n(t, x)-t / \bar{l}| \leq|n(t, x)|+t / \bar{l} \leq A t$ for some constant $A$. Now the left-hand side of (18) does not exceed I + II where

$$
\begin{aligned}
\mathrm{I} & :=\int_{\mid\left(n(t, x)-t / \bar{\eta} \leq t^{3 / 4}\right.} \max _{m \leq t^{3 / 4}}\left(\sum_{i \leq m} F\left(T^{i} x\right)\right)^{2} d \mu(x), \\
& \ll t^{\frac{3}{4}}=o(t)
\end{aligned}
$$

by (19) and Hölder's inequality, and where

$$
\begin{aligned}
\mathrm{II} & :=\int_{|n(t, x)-t / \bar{l}|>t^{3 / 4}} \max _{m \leq A t}\left(\sum_{i \leq m} F\left(T^{i} x\right)\right)^{2} d \mu(x) \\
& \leq\left(\mu\left(|n(t, x)-t / \bar{l}|>t^{\frac{3}{4}}\right)\right)^{\delta /(2+\delta)} \\
& \cdot\left(E_{\mu} \max _{m \leq A t}\left|\sum_{i \leq m} F\left(T^{i} x\right)\right|^{2+\delta}\right)^{2 /(2+\delta)} \\
& \ll t^{\left(1+\frac{1}{2} \delta\right) 2 /(2+\delta)} o(1)=o(t)
\end{aligned}
$$

by (19) and lemma 3 . This proves (18). Hence by proposition 1 there exists a standard Brownian motion $(B(t), t \geq 0)$ such that

$$
\sum_{i \leq n} F\left(T^{i} x\right)-B(\tau n) \ll n^{\frac{1}{2}-\lambda}
$$

for $\mu$-almost all $x$. Hence for $\mu$-almost all $x$

$$
\sum_{i \leq n(t, x)} F\left(T^{i} x\right)-B(\tau n(t, x)) \ll(n(t, x))^{\frac{1}{2}-\lambda} \text {. }
$$

Now by the argument in $[5$, p. 24]

$$
B(\tau n(t, x))-B\left(\sigma^{2} t\right) \ll t^{\frac{1}{2}-\lambda}
$$

for $\mu$-almost all $x$. Since $n(t, x) \ll t$ the result for the approximation with respect to $\mu$ follows from (20), (21) and lemma 2 .

In order to prove the approximation in (2) when $B$ is replaced by a standard Brownian motion on the probability space $\left(\Sigma, \mu^{*}\right)$, where $d \mu^{*}(x)=\bar{l}^{-1} l(x) d \mu(x)$, first note that all estimates which hold $\mu$-a.e. also hold $\mu^{*}$-a.e. and conversely because both probabilities are equivalent. Hence lemmas 2 and 3 hold $\mu^{*}$-a.e. Also the arguments in (18)-(21) remain valid since for an integrable function $g$

$$
\int g d \mu \ll \int g d \mu^{*} \ll \int g d \mu .
$$

All that remains to show is that proposition 1 can still be applied.

We first show that the coordinate process $\left\{\xi_{n}, n \in \mathbb{Z}\right\}$ is still $\psi$-mixing with an exponentially fast decreasing rate. We shall make use of the following lemma which is easily proved by approximation by simple functions.

LEMMA 4. If $\xi$ and $\eta$ are integrable and $\xi$ is measurable with respect to $\mathscr{A}$ and $\eta$ is measurable with respect to $\mathscr{B}$ then

where

$$
|E \xi \eta-E \xi E \eta| \leq \psi(n) E|\xi| E|\eta|
$$

$$
\psi(n):=\sup \left\{\left|\frac{P(A \cap B)}{P(A) P(B)}-1\right|: A \in \mathscr{A}, B \in \mathscr{B}\right\}
$$


Let $A \in(\tilde{\gamma})_{-m}^{k}, B \in(\tilde{\gamma})_{k+4 n}^{k+m^{\prime}}$ and assume first that $k \geq-2 n$. The case $k<-2 n$ can be treated similarly. Then by lemma 4

$$
\begin{aligned}
\left|\mu^{*}(A \cap B)-\mu^{*}(A) \mu^{*}(B)\right| \\
\leq \bar{l}^{-1} \int\left|l(x)-E_{\mu}\left(l \mid(\tilde{\gamma})_{-n}^{n}\right)(x)\right| 1_{A \cap B}(x) d \mu(x) \\
\quad+\bar{l}^{-1}\left|\int_{A \cap B} E_{\mu}\left(l \mid(\tilde{\gamma})_{-n}^{n}\right) d \mu-\int_{A} E\left(l \mid(\tilde{\gamma})_{-n}^{n}\right) d \mu \mu(B)\right| \\
+\bar{l}^{-1} \int_{A}\left|E_{\mu}\left(l \mid(\tilde{\gamma})_{-n}^{n}\right)-l\right| d \mu \mu(B) \\
+\mu^{*}(A)\left|\mu(B)-\bar{l}^{-1} \int_{B} E_{\mu}\left(l \mid(\tilde{\gamma})_{-n}^{n}\right) d \mu\right| \\
+\mu^{*}(A) \bar{l}^{-1} \int_{B}\left|l-E_{\mu}\left(l \mid(\tilde{\gamma})_{-n}^{n}\right)\right| d \mu \ll \beta^{* n} \mu^{*}(A) \mu^{*}(B)
\end{aligned}
$$

where $\beta^{*}<1$, since $\left\{\xi_{n}\right\}$ is $\psi$-mixing with respect to $\mu$. We will apply proposition 1 to the sequence $\eta_{n}^{*}=F T^{n}-\int F T^{n} d \mu^{*}(n \in \mathbb{Z})$. Observe that $\left(\eta_{n}^{*}\right)$ need no longer be a stationary sequence and that $\sum_{i \leq n} \eta_{i}^{*}-\sum_{i \leq n} F T^{i} \ll 1$, since by (14)

$$
\begin{aligned}
\left|\int F \circ T^{n} d \mu^{*}\right| \leq & \bar{l}^{-1}\left|\int F T^{n}\left(l-E_{\mu}\left(l \mid(\tilde{\gamma})_{-[n / 3]}^{[n / 3]}\right)\right) d \mu\right| \\
& +\bar{l}^{-1} \int E_{\mu}\left(l \mid(\tilde{\gamma})_{-[n / 3]}^{[n / 3]}\right)\left|F T^{n}-E_{\mu}\left(F T^{n} \mid(\tilde{\gamma})_{n-[n / 3]}^{n+[n / 3]}\right)\right| d \mu \\
& +\bar{l}^{-1}\left|\int E_{\mu}\left(l \mid(\tilde{\gamma})_{-[n / 3]}^{[n / 3]}\right) E_{\mu}\left(F T^{n} \mid(\tilde{\gamma})_{n-[n / 3]}^{n+[n / 3]}\right) d \mu\right| \\
\ll & \alpha^{\frac{1}{3} n}+\left(\int \mid F-E_{\mu}\left(F\left|(\tilde{\gamma})_{-[n / 3]}^{[n / 3]}\right|^{2+\delta} d \mu\right)^{1 / 2+\delta}\right. \\
\ll & n^{-(2+(7 / \delta))}
\end{aligned}
$$

and hence $\sum_{n \geq 0}\left|\int F T^{n} d \mu^{*}\right|<\infty$. Conditions (10) and (11) are trivially satisfied. It remains to verify (12).

Fix $n \geq 1$ and let $m \leq 3 n / 4$. Note that there is a $q<1$ such that for all $C \in(\tilde{\gamma})_{m-n}^{m+n}$

$$
\begin{aligned}
& \left|\int_{C} F\left(T^{m} y\right) \bar{l}^{-1} l(y) d \mu(y) \mu(C)-\int_{C} F\left(T^{m} y\right) d \mu(y) \mu^{*}(C)\right| \\
& \quad=\left|\iint_{C \times C} \bar{l}^{-1} F\left(T^{m} y\right)(l(y)-l(z)) d \mu(y) d \mu(z)\right| \\
& \quad \ll q^{n} \mu(C) \int_{C}\left|F\left(T^{m} y\right)\right| d \mu(y),
\end{aligned}
$$


since for $y, z \in C$ and $|i|<n / 4, y_{i}=z_{i}$. Hence

$$
\begin{aligned}
\left\{E_{\mu^{*}} \mid\right. & \left.F T^{m}-\left.E_{\mu^{*}}\left(F T^{m} \mid(\tilde{\gamma})_{m-n}^{m+n}\right)\right|^{2+\delta}\right\}^{1 /(2+\delta)} \\
= & \left\{\sum_{C \in(\tilde{\gamma})_{m-n}^{m+n}} \int_{C}(l(x) / \bar{l})\left[\mu^{*}(C) \bar{l}\right]^{-2-\delta} \mid F\left(T^{m} x\right) \mu^{*}(C)\right. \\
& \left.-\left.\int_{C} F\left(T^{m} y\right)(l(y) / \bar{l}) d \mu(y)\right|^{2+\delta} d \mu(x)\right\}^{1 /(2+\delta)} \\
\ll & q^{n}\left\{E_{\mu^{*}}\left[E_{\mu}\left(\left|F T^{m}\right|^{2+\delta} \mid(\tilde{\gamma})_{m-n}^{m+n}\right)\right]\right\}^{1 /(2+\delta)} \\
& +\left\{\sum_{C} \int_{C}(l(x) / \bar{l})\left|F\left(T^{m} x\right)-(1 / \mu(C)) \int_{C} F\left(T^{m} y\right) d \mu(y)\right|^{2+\delta} d \mu(x)\right\}^{1 /(2+\delta)} \\
\ll & q^{n}\left(E_{\mu}\left(\left|F T^{m}\right|^{2+\delta}\right)\right)^{1 /(2+\delta)}+n^{-(2+(7 / \delta))} \ll n^{-(2+(7 / \delta))} .
\end{aligned}
$$

Now let $m>3 n / 4$. First note that by elementary properties of conditional expectations, Minkowski's inequality and the conditional Hölder inequality

$$
\begin{aligned}
& \left\{E_{\mu^{*}}\left(\left|F T^{m}-E_{\mu^{*}}\left(F T^{m} \mid(\tilde{\gamma})_{m-n}^{m+n}\right)\right|^{2+\delta}\right)\right\}^{1 /(2+\delta)} \\
& \quad \leq 2\left\{E_{\mu^{*}}\left(\left|F T^{m}-E_{\mu^{*}}\left(F T^{m} \mid(\tilde{\gamma})_{m-[n / 4]}^{m+[n / 4]}\right)\right|^{2+\delta}\right)^{1 /(2+\delta)}\right.
\end{aligned}
$$

For simplicity we put $k:=[n / 4]$. Using lemma 4 twice we obtain for $x \in C \in(\tilde{\gamma})_{m-k}^{m+k}$ :

$$
\begin{aligned}
& \left|E_{\mu^{*}}\left(F T^{m} \mid(\tilde{\gamma})_{m-k}^{m+k}\right)(x)-E_{\mu}\left(F T^{m} \mid(\tilde{\gamma})_{m-k}^{m+k}\right)(x)\right| \\
& =\left|\frac{1}{\mu^{*}(C)} \int_{C} F\left(T^{m} y\right) \frac{l(y)}{T} d \mu(y)-\frac{1}{\mu(C)} \int_{C} F\left(T^{m} y\right) d \mu(y)\right| \\
& \leq \frac{1}{\mu^{*}(C)}\left\{\int_{C} 1_{C}(y)\left|F\left(T^{m} y\right)-E_{\mu}\left(F T^{m} \mid(\tilde{\gamma})_{m-k}^{m+k}\right)(y)\right| \frac{l(y)}{\bar{l}} d \mu(y)\right. \\
& +\int_{C}(y)\left|E_{\mu}\left(F T^{m} \mid(\tilde{\gamma})_{m-k}^{m+k}\right)(y)\right| \bar{l}^{-1}\left|l(y)-E_{\mu}\left(l \mid(\tilde{\gamma})_{-k}^{k}\right)(y)\right| d \mu(y) \\
& +\mid \int_{C}(y) E_{\mu}\left(F T^{m} \mid(\tilde{\gamma})_{m-k}^{m+k}\right)(y) \bar{l}^{-1} E_{\mu}\left(l \mid(\tilde{\gamma})_{-k}^{k}\right)(y) d \mu(y) \\
& \left.\quad-\int_{C} F\left(T^{m} y\right) d \mu(y) \mid\right\}^{m} \\
& +\int_{C}\left|F\left(T^{m} y\right)\right| d \mu(y)\left[\mu^{*}(C) \mu(C)\right]^{-1} \\
& \times\left\{\left|\mu(C)-\int_{C} \bar{l}^{-1} E_{\mu}\left(l \mid(\tilde{\gamma})_{-k}^{k}\right)(y) d \mu(y)\right|\right. \\
& \left.+\int_{C} \bar{l}^{-1}\left|l(y)-E_{\mu}\left(l \mid(\tilde{\gamma})_{-k}^{k}\right)(y)\right| d \mu(y)\right\}
\end{aligned}
$$

$\ll\left(E_{\mu}\left(\left|F T^{m}-E_{\mu}\left(F T^{m} \mid(\tilde{\gamma})_{m-k}^{m+k}\right)\right| \mid(\tilde{\gamma})_{m-k}^{m+k}\right)(x)+\beta^{n / 4}+q^{n}\right) E_{\mu}\left(\left|F T^{m}\right| \mid(\tilde{\gamma})_{m-k}^{m+k}\right)(x)$. 
Here we have used twice that $\left\{\xi_{n}\right\}$ is $\psi$-mixing with respect to $\mu$. Integrating over $x$ with respect to $\mu^{*}$ we obtain by Minkowski's inequality

$$
\begin{aligned}
\left(E_{\mu^{*}} \mid\right. & \left.F T^{m}-\left.E_{\mu^{*}}\left(F T^{m} \mid(\tilde{\gamma})_{m-k}^{m+k}\right)\right|^{2+\delta}\right)^{1 /(2+\delta)} \\
\leq & \left(E_{\mu^{*}}\left|F T^{m}-E_{\mu}\left(F T^{m} \mid(\tilde{\gamma})_{m-k}^{m+k}\right)\right|^{2+\delta}\right)^{1 /(2+\delta)} \\
& +\left(E_{\mu^{*}}\left|E_{\mu^{*}}\left(F T^{m} \mid(\tilde{\gamma})_{m-k}^{m+k}\right)-E_{\mu}\left(F T^{m} \mid(\tilde{\gamma})_{m-k}^{m+k}\right)\right|^{2+\delta}\right)^{1 /(2+\delta)} \\
\ll & n^{-\left(2+7 \delta^{-1}\right)}+\left(\beta^{n / 4}+q^{n}\right)\left(E_{\mu}\left(E_{\mu}\left(\left|F T^{m}\right| \mid(\tilde{\gamma})_{m-k}^{m+k}\right)\right)^{2+\delta}\right)^{1 /(2+\delta)} \\
& +\left(E_{\mu}\left|F T^{m}-E_{\mu}\left(F T^{m} \mid(\gamma)_{m-k}^{m+k}\right)\right|^{2+\delta}\right)^{1 /(2+\delta)} \\
\ll & n^{-\left(2+7 \delta^{-1}\right)} .
\end{aligned}
$$

Note that the constants implied by $\ll$ do not depend on $m$ and $n$. Hence relation (12) follows. Similarly condition (13) can be verified. This completes the proof of the theorem.

\section{REFERENCES}

[1] P. Billingsley. Convergence of Probability Measures. J. Wiley: New York, 1968.

[2] R. Bowen. Equilibrium States and the Ergodic Theory of Anosov Diffeomorphisms. Lecture Notes in Math. 470. Springer: Berlin-Heidelberg-New York, 1975.

[3] J. L. Doob. Stochastic Processes. J. Wiley: New York, 1953.

[4] I. A. Ibragimov. Some limit theorems for stationary processes. Theor. Probability Appl. 7 (1962) 349-382.

[5] W. Philipp \& W. Stout. Almost sure invariance principles for partial sums of weakly dependent random variables. Mem. Amer. Math. Soc. 161 (1975).

[6] M. Ratner. The central limit theorem for geodesic flows on n-dimensional manifolds of negative curvature. Israel J. Math. 16 (1973), 181-197.

[7] A. Rényi. Wahrscheinlichkeitsrechnung. Deutscher Verlag Wissensch.: Berlin, 1962.

[8] D. Ruelle, Statistical mechanics of a one-dimensional lattice gas. Commun. Math. Phys. 9 (1968), 267-278.

[9] R. J. Serfling, Moment inequalities for the maximum cumulative sum. Ann. Math. Stat. 41 (1970), 1227-1234

[10] Y. G. Sinai, Gibbs measures in ergodic theory. Uspeki Mat. Nauk (4) 27 (1972), 21-63. 